\title{
Neural Network based - Arrhythmia Monitoring Device: A Pivotal Clinical Trial
}

\author{
Ardian Rizal, Puspa Lestari, Satria Mandala, Budi Satrijo, Sasmojo Widito,
}

\begin{abstract}
Arrhythmia or irregular heart beat had wide range of clinical manifestations, from benign arrhythmia that not need any medication to life threatening condition. It can occur permanently or intermittently. Intermittent arrhythmia needs specific diagnostic tools that can record the electrocardiogram continuously. This research was sought to analysed the sensitivity, specificity, Positive Predictive Value, and Negative Predictive Value of arrhythmia monitoring device that based on neural network based artificial intelligent. The pivotal clinical trial was involved a total 103 people (health and stable arrhythmia patients). This research used a diagnostic test by comparing the electrocardiography (ECG) from prototype with standard ECG for diagnose arrhythmia. The Arrhythmia Monitoring System that we developed has three hardware components; smartphones, server for arrhythmia detection and patchable ECG recorder. All three components are connected with internet of things (IoT) technology. The architecture of Arrhythmia software monitoring included ECG signals pre-processing, beats detection, features extraction for detecting $V T / V F$, and classification for detecting $V T / V F$. Features extraction such as heart rate variability $(H R V)$ and $T$ wave alternans. We compared the ECG of arrhythmia prototype monitoring device with standard Holter monitoring. We enrolled 103 patients. There was no significant difference of heart rate between arrhythmia prototype monitoring device and standard Holter $(87.26 \pm 11.2$ vs $86.07 \pm 9.15, P=0.43)$. There was significant different of maximum and minimum heart rate between arrhythmia prototype monitoring device and standard holter monitoring (121.3 \pm 31.7 vs $131.0 \pm 10.8, p=0.000$, and $65.1 \pm 13.5$ vs $73.07 \pm 10.02, p=0.000)$. This device has low sensitivity $80 \%(95 \%$ CI 75\% - 82\%) and high specificity 91.8\% (95\% CI 85\%-92\%) for detecting the abnormal ECG. The Positive Predictive Value (PPV) was 63.2\% (95\% CI 58.8\% - 67.52\%) and Negative Predictive Value (NVP) was $96.3 \%$ (95\% CI 94.7\% - 98.3\%). This device demonstrates an ability to detect PVC and PAC (Sensitivity $71.4 \%$ (95\% CI 66.4\% - 76.4\%) and 75\% (95\% CI 72\%-78\%), Specificity 97.8\% (95\% CI 95.8-99.8\%) and 91.7\% (95\% CI 83.4\%99.7\%, respectively). The PPV of this device to detect PVC and PAC was $71.4 \%$ (95\% CI 66.4\%-76.4\%) and $72.7 \%$. (95\% CI 68.7\%-76.7\%) The NPV of this device to detect PVC and PAC was 97.8\% (95\% CI 95.8\%-99.85) and 98.9\% (95\% CI 98.1\%-99.7\%), respectively. This study found that the device to be a valuable diagnostic tool that has relatively low sensitivity but high specificity for diagnosing Abnormal ECG, PVC and PAC. According to the results of our study, we found that the device to be a valuable diagnostic tool that has relatively high sensitivity and specificity for diagnosing Abnormal ECG, PVC and PAC.
\end{abstract}

Keyword: Arrhythmia; Arrhythmia Monitoring Device; Holter Monitoring

Revised Manuscript Received on January 2, 2020.

* Correspondence Author

Ardian Rizal, Cardiology and Vascular Medicine Department, Faculty

of Medicine, Universitas Brawijaya, drardianrizal@ub.ac.id

Puspa Lestari*, Cardiology and Vascular Medicine Department,

Faculty of Medicine, Universitas Brawijaya. pusparyath@gmail.com

Satria Mandala, Telkom University.

Budi Satrijo, Cardiology and Vascular Medicine Department, Faculty of Medicine, Universitas Brawijaya.

Sasmojo Widito, Cardiology and Vascular Medicine Department,

Faculty of Medicine, Universitas Brawijaya.

\section{INTRODUCTION}

Cardiac arrhythmia is the condition where the heart beats irregularly. It clinical features ranges from asymptomatic condition to devastating condition such as stroke and even sudden cardiac death (Jordaens 2018). Lethal arrhythmia such as Ventricular Tachycardia (VT) and Ventricular Fibrillation (VF) has contributed to sudden cardiac death cases (Kobayashi 2018). While Atrial Fibrillation (AF) is type of arrhythmia that often found in hypertension and heart failure patients, and also the risk factor of stroke (Rizal and Yuniadi 2019). Since it used to appear intermittently, ambulatory electrocardiography (ECG) monitoring is one of the diagnostic tools to be used in current clinical practice.

Cardiac arrhythmia also had huge contribution to health spending in Indonesia. According to Indonesian National Insurance Agency (BPJS KESEHATAN) data, cardiovascular problem contributes to nearly ten trillion rupiah spending in 2019. The biggest spending among another health problem in Indonesia. One thing that maybe blamed in that condition is the dependency of imported drug and health equipment.

Since 2017 we had developed prototype of Arrhythmia Monitoring System. The prototype is mainly an ECG recorder, with additional features such as cloud computing and data storing, neural network-based arrhythmia recognition, smartphone and web-based monitoring system and online warning system that can sent information directly to the physician through mobile data. Arrhythmia detection algorithm and phase 1 clinical trial had been performed before. The current arrhythmia prototype monitoring device produce single lead ECG recording. In the future we will upgrade to triple lead to enhance the diagnostic accuracy.

Before the device can be clinically used, several steps of trials had to be conducted. This trial is a pivotal clinical trial to test the accuracy and the performance of the device as an arrhythmia detections tool.

\section{MATERIAL AND METHODS}

\section{A. Study Design}

Patient that being hospitalized in the Cardiovascular Centers ward of Saiful Anwar General Hospital was recruited consecutively. The exclusion criteria were patients that refused to joined the research and the patients that clinically in unstable condition, determined by the attending physician. All of those patients were simultaneously monitored by standard holter monitoring device (BTL Cardio Point H-600) and prototype device. The ECG recording duration was 10 minutes for each monitoring device (BTL and arrhythmia prototype device). 


\section{B. ECG Interpretation}

The results of both ECG recording were analyzed separately. The standard holter device (BTL Cardio Point H-600) was analyzed by two cardiologists from Saiful Anwar General Hospital. While, the ECG recording from this device was extracted automatically rom the cloud data and managed by technician from Telkom University. Several ECG characteristics were analyzed from both devices. Maximum heart rate (HR), minimum HR, number of Premature Ventricular Contraction (PVC), number of Premature Atrial Contraction (PAC), number of Atrial Fibrillation (AF) episodes and number of Ventricular Tachycardia (VT) or Ventricular Fibrillation (VF) are the parameters that are being measured.

\section{Statistical Analysisis}

Statistical analysis was conducted using SPSS (version 16.0, International Business Machines, Armonk, New York, USA). Continuous data were summarized as mean \pm SD. Comparisons of characteristics and heart rate data between arrhythmia prototype monitoring device groups and standard Holter groups were performed using independent $t$ tests for normally distributed data, Mann-Whitney $U$ tests for non-normally distributed data, and chi-square tests for categorical data. The goodness of fit was evaluated by use of the $\mathrm{x} 2$ test. The null hypothesis (Ho) was "the patient has abnormal ECG." When Ho was actually true but incorrectly rejected, a type I error or a false positive occurred. When Ho was actually false but incorrectly accepted, a type II error or a false negative occurred. For a given ABD-EF threshold, the probability of making type I errors $(\alpha)$ and the probability of making type II errors $(\beta)$ were determined from the probability density functions of positive and negative data, respectively. Sensitivity, or true positive rate, was determined by Sensitivity $=1-\alpha$ Specificity, or true negative rate, was determined by Specificity $=1-\beta$. Figure 1 presents ECG recording and rhythm determinations using arrhythmia prototype monitoring device. First, the incoming digitized ECG samples are segmented into heartbeats and their $\mathrm{RR}$ interval features. The segmentation is performed based on detecting the $\mathrm{R}$ peak to determine the normal and abnormal rhythm.

Table 1. Differences Heart Rate Using Arrhythmia Prototype Monitoring Device and Standard Holter

\begin{tabular}{|c|c|c|c|}
\hline Variable & Arrhythmia Prototype Monitoring Device & Standard Holter & P \\
\hline Mean of Heart Rate & $87.26 \pm 11.2$ & $86.07 \pm 9.15$ & 0.43 \\
\hline Maximum of Heart Rate & $121.3 \pm 31.7$ & $131.0 \pm 10.8$ & 0.000 \\
\hline Minimum of Heart Rate & $65.1 \pm 13.5$ & $73.07 \pm 10.02$ & 0.000 \\
\hline
\end{tabular}

In
$+\stackrel{\text { Dashboard }}{\text { Add Account }}$ Add Doctor / Nurse

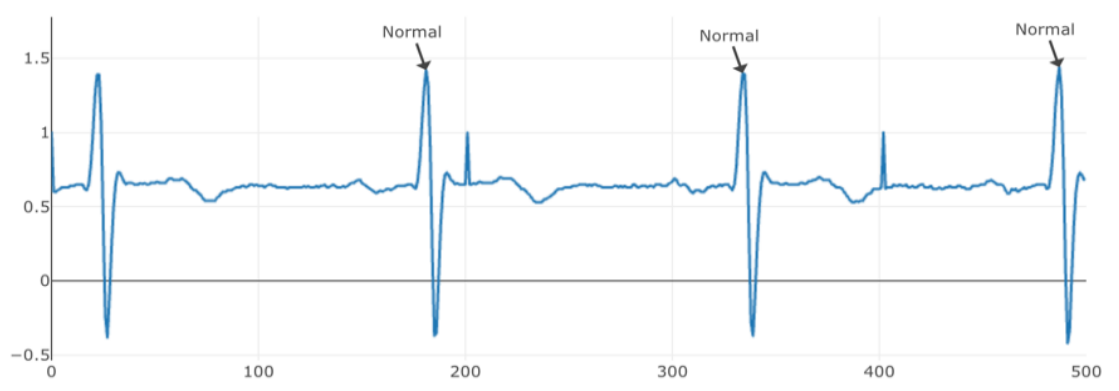

Figure 1. ECG recording and rhythm determination using arrhythmia prototype monitoring device

Table 2. Sensitivity, Specificity, PPV and NPV of Diagnosing Abnormal ECG, PVC and PAC

\begin{tabular}{|l|l|l|l|}
\hline & Abnormal ECG & PVC & PAC \\
\hline Sensitivity & $80 \%$ & $71.4 \%$ & $75 \%$ \\
\hline Specificity & $91.8 \%$ & $97.8 \%$ & $91.7 \%$ \\
\hline PPV (Positive Predictive Value) & $63.2 \%$ & $71.4 \%$ & $72.7 \%$ \\
\hline NPV (Negative Predictive Value) & $96.3 \%$ & $97.8 \%$ & $98.9 \%$ \\
\hline
\end{tabular}




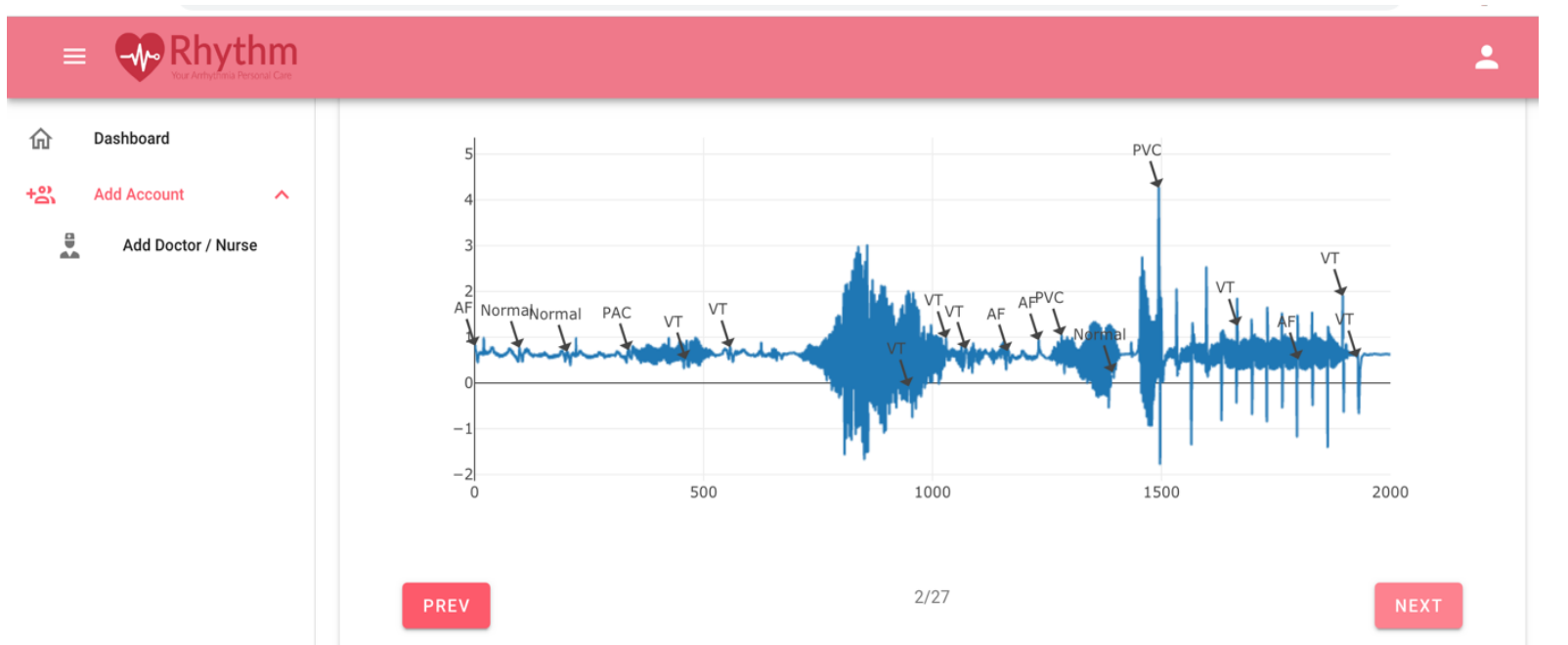

Figure 2. ECG Waveforms and Artifacts

\section{RESULT AND DISCUSSION}

\section{A. Result}

Total 103 patients were recruited. All of the patient had dual ECG recording data that had been recorded simultaneously. The mean age of this subjects were $33.36 \pm$ $8.68,64 \%$ were male and $36 \%$ were female.

There was no significant difference of heart rate between arrhythmia prototype monitoring device and standard holter $(87.26 \pm 11.2$ vs $86.07 \pm 9.15, \mathrm{P}=0.43)$. It showed that the subjects within the groups are very similar. There was significant different of maximum and minimum heart rate between arrhythmia prototype monitoring device and standard holter monitoring $(121.3 \pm 31.7$ vs $131.0 \pm 10.8, \mathrm{p}=$ 0.000 , and $65.1 \pm 13.5$ vs $73.07 \pm 10.02, \mathrm{p}=0.000$ ). The data showed that arrhythmia prototype monitoring device relied upon when assessing averaged of heart rate.

Arrhythmia prototype monitoring device has low sensitivity $80 \%(95 \%$ CI $75 \%-82 \%)$ and high specificity $91.8 \%(95 \%$ CI $85 \%$ - 92\%) for detecting the abnormal ECG. The Positive Predictive Value (PPV) was $63.2 \%$ (95\% CI 58.8\% - 67.52\%) and Negative Predictive Value (NVP) was 96.3\% (95\% CI 94.7\% - 98.3\%). Arrhythmia prototype monitoring device demonstrate an ability to detect PVC and PAC (Sensitivity 71.4\% (95\% CI 66.4\% - 76.4\%) and $75 \%$ (95\% CI 72\%-78\%), Specificity $97.8 \%$ (95\% CI $95.8-99.8 \%)$ and $91.7 \% \quad(95 \%$ CI $\quad 83.4 \%-99.7 \%$, respectively). The PPV of Arrhythmia prototype monitoring device to detect PVC and PAC was $71.4 \%$ (95\% CI $66.4 \%$ $76.4 \%$ ) and $72.7 \%$. (95\% CI 68.7\%-76.7\%) The NPV of this device to detect PVC and PAC was $97.8 \%$ (95\% CI 95.8\%99.85) and $98.9 \%$ (95\% CI 98.1\%-99.7\%), respectively. This study found that Arrhythmia prototype monitoring device to be a valuable diagnostic tool that has relatively low sensitivity but high specificity for diagnosing Abnormal ECG, PVC and PAC.

\section{B. Discussion}

Arrhythmia prototype monitoring device to be a valuable diagnostic tool that has relatively low sensitivity but high specificity for diagnosing Abnormal ECG, PVC and PAC. Noise and artefact in ECG recording are something that almost cannot be avoided. The patient movement, local muscle contraction and electrical interference are some causes of noise and artefact in the recording process (Rodrigues, Belo, and Gamboa 2017). In automatic ECG signal labeling depends mainly on the algorithm and the quality of the recording (Imtiaz et al. 2016). ECG recording artefact can be misinterpreted with fast heart rate (due to narrow peak $\mathrm{R}$ to $\mathrm{R}$ interval) and even arrhythmia (such as VT, VF and AF). The reason why this device had difficulty in determining the minimum and maximum heart rate because of recording artefact. In the single lead ECG differentiating recording artefact with arrhythmia is almost impossible (Samaniego, Morris, and Brady 2003). In the other hand, Arrhythmia prototype monitoring device had no artefact detection algorithm. The machine learning didn't have enough example of ECG artefact. In the clinical use, every automatic signal labelling still needs to be validated by the physician. Another way to be more sophisticated ECG signal auto labelling is by adding another ECG lead (multi lead ECG recording).

The accuracy of Arrhythmia prototype monitoring device in detecting another type of arrhythmia such as AF, VT and VF cannot be evaluated in this study due to very limited sample. The exclusion criteria that excluded patients with unstable clinical condition may contribute to that limited samples of current arrhythmia. As those type of arrhythmia's prevalence is higher in the acute unstable clinical condition. In the future, trials that involving those type of patient had to be conducted to evaluate the accuracy.

\section{CONCLUSION}

This study has found the Arrhythmia prototype monitoring device to be a valuable diagnostic tool that has relatively low sensitivity but high specificity for diagnosing Abnormal ECG, PVC and PAC. This test can use for ruling in abnormal ECG, PVC, and PAC. Further studies and improvement are also needed to increase the diagnostic accuracy. 


\section{REFERENCES}

1. Imtiaz, Syed Anas, James Mardell, Siavash Saremi-Yarahmadi, and Esther Rodriguez-Villegas. 2016. "ECG Artefact Identification and Removal in MHealth Systems for Continuous Patient Monitoring." Healthcare Technology Letters 3 (3): 171-76. https://doi.org/10.1049/htl.2016.0020.Jordaens, L. 2018. “A Clinical Approach to Arrhythmias Revisited in 2018." Netherlands Heart Journal 26 (4): 182-89. https://doi.org/10.1007/s12471-018-1089-1.

2. Kobayashi, Yoshinori. 2018. "How to Manage Various Arrhythmias and Sudden Cardiac Death in the Cardiovascular Intensive Care." Journal of Intensive Care 6: 23. https://doi.org/10.1186/s40560-0180292-x.

3. Rizal, Ardian, and Yoga Yuniadi. 2019. "Epigenetic Implication in Atrial Fibrillation: A Potential Biomarker?" Journal of Laboratory and Precision $\quad 3$ Medicine http://jlpm.amegroups.com/article/view/5128.

4. Rodrigues, João, David Belo, and Hugo Gamboa. 2017. "Noise Detection on ECG Based on Agglomerative Clustering of Morphological Features." Computers in Biology and Medicine 87 (August):

$322-34$. https://doi.org/10.1016/j.compbiomed.2017.06.009.

5. Samaniego, N. C., F. Morris, and W. J. Brady. 2003. "Electrocardiographic Artefact Mimicking Arrhythmic Change on the ECG.” Emergency Medicine Journal 20 (4): 356-57. https://doi.org/10.1136/emj.20.4.356.

\section{AUTHORS PROFILE}

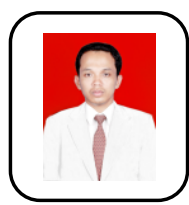

Ardian Rizal, MD, FIHA

- Staff of Departement of Cardiology and Vascular Medicine, Saiful Anwar Teaching Hospital, and School of Medicine University of Brawijaya, Malang, Indonesia

- Chief of Division of Arrhythmia, Pacing, and Electrophysiology

- The research interest on atrial fibrillation and cardiac arrhythmia

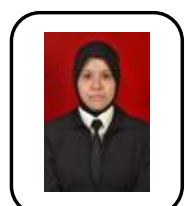

Puspa Lestari, MD

- Resident of Cardiology and Vascular Medicine, Saiful Anwar Teaching Hospital, and School of Medicine University of Brawijaya, Malang, Indonesia

- The research interest on cardiac arrhythmia

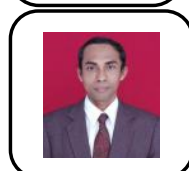

Satria Mandala, Ph.D

- Lecture at Telkom University

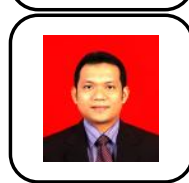

Budi Satrijo, MD, FIHA

- Staff of Departement of Cardiology and Vascular Medicine, Saiful Anwar Teaching Hospital, and School of Medicine University of Brawijaya, Malang, Indonesia

- Chief of Integrated Heart Service Center, Saiful Anwar Teaching Hospital, and School of Medicine University of Brawijaya, Malang, Indonesia

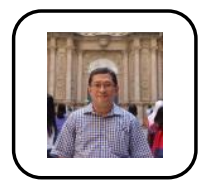

Sasmojo Widito, MD, FIHA, FAsCC, FAPSIC

- Head of Departement of Cardiology and Vascular Medicine, Saiful Anwar Teaching Hospital, and School of Medicine University of Brawijaya, Malang, Indonesia

- Chief of division of Interventional Cardiovascular, Departement of Cardiology and Vascular Medicine, Saiful Anwar Teaching Hospital, and School of

Medicine University of Brawijaya, Malang, Indonesia

- The research interest include:

- Research on behavior of vascular systems

- Research on behavior of cardiac electricity systems 